Accretion Phenomena and Related Outflows, IAU Colloquium 163

ASP Conference Series, Vol. 121, 1997

D.T. Wickramasinghe, L. Ferrario, and G.V. Bicknell, eds.

\title{
X-Ray Observations Of SS 433 With ASCA
}

\author{
T. Kotani, N. Kawai, and M. Matsuoka
}

Cos. Rad. Lab., RIKEN, 2-1 Hirosawa, Wako, 351-01 Japan

\author{
W. Brinkmann \\ Max-Planck-Institut für Extraterrestrische Physik, Giessenbachstrasse, \\ D-85740 Garching, Germany
}

\begin{abstract}
SS 433 has been observed with ASCA for three years since the launch in 1993. The excellent energy resolution of ASCA revealed the Doppler-shifted emission lines from the both jets, though it had been generally accepted that the X-ray emitting region of the receding jet is hidden behind the accretion disk. Thus the estimation on the properties of the X-ray jet, such as length, temperature, and mass outflow rate, should be revised in accordance with the ASCA data. Modeling the jet as a radiating ballistic plasma, we determined these quantities. It is also tried to determine the mass of the system from a data covering an eclipse.
\end{abstract}

\section{Introduction}

SS 433 is an enigmatic binary with a relativistic, precessing bipolar jet. Though it has been studied for more than fifteen years since its discovery, some of the fundamental physics of the system, such as the mechanism of the acceleration and the precession, the nature of the compact object, still remain unknown.

The excellent spectral resolution of the SIS on ASCA (Tanaka et al. 1994) is suitable for studies of the complex X-ray spectra of SS 433 . Here we report the preliminary results from the ASCA observations conducted at various precessional and orbital phases.

\section{Modeling the Jet}

In figure 1, an example of spectra is shown. As seen, it is abundant in pairs of Doppler-shifted emission lines from both of the two oppositely directed jets. The pairs of $\mathrm{Si}, \mathrm{S}, \mathrm{Ar}$, etc. are also seen in the lower-energy band (Kotani et al. 1994). That is contradictory to the previous general consensus that the $\mathrm{X}$-ray-emitting region of the jet is short so that receding one is always occulted by the disk (Watson et al. 1986; Stewart et al. 1987). It was concluded from the ASCA data that the X-ray jet must be longer than the previous estimations of $\lesssim 12 \mathrm{~cm}$.

Together with the observed flux, a model spectrum is plotted in figure 1. The jet was modeled as a conical plasma cooled by expansion and X-ray radiation 


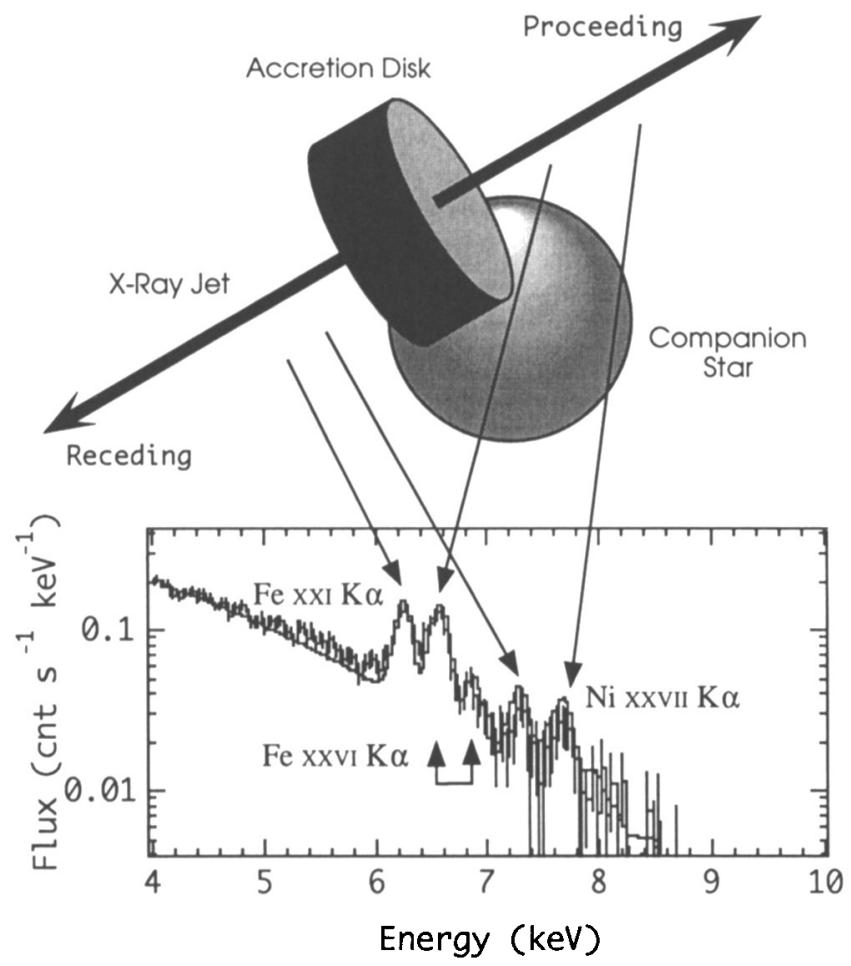

Figure 1. A Spectrum of SS 433 taken with ASCA SIS. Pairs of Doppler-shifted emission lines of Fe XXV K $\alpha$, Fe XXVI K $\alpha$, and Ni XXVII $\mathrm{K} \alpha$ are clearly seen. They are evidence of the visibility of both X-ray jets, thus the X-ray jets must be longer than the previous estimations.

(Brinkmann et al. 1991; Kotani et al. 1996a). The principal input parameters are the mass outflow rate per jet $\dot{m}$, the initial electron density at the base $n_{\mathrm{e} 0}$, the initial temperature $T_{0}$, and the opening half angle $\Theta$ of the cone. The abundances are also important parameters which affect the resultant emission line flux considerably. It was found that the jet plasma is rich in some metals (Kotani et al. 1996b). Especially, $\mathrm{Ni}$ is as abundant as a few tens of the solar value. Because $\mathrm{Ni}$ is produced in supernovae and the jet matter originates in the envelope of the companion star, the overabundance of Ni may be an evidence of the peculiar history of the system. Since some radiative-acceleration mechanisms are predicted to be sensitive to the abundance of the plasma (Shapiro et al. 1986), Ni might play an important role in the acceleration of the jet, .

From the line flux ratio of Fe XXVI $\mathrm{K} \alpha$ and Fe XXv $\mathrm{K} \alpha$, the base temperature was determined to be $21 \pm 3 \mathrm{keV}$ (Kotani et al. 1996a). The temperature determined by the Fe line flux ratio is most reliable since it is almost free from other uncertainties such as the gain and the efficiency of the detector, or other input parameters. Even if the assumed abundance of iron was wrong, the resultant temperature would not be affected much. 


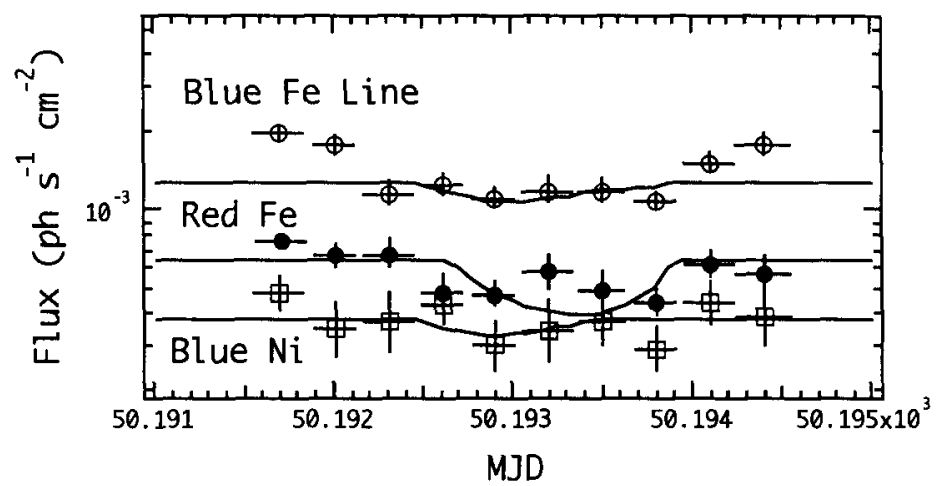

Figure 2. Emission-line light curves. The open circles correspond to the sum of the blue-shifted Fe lines and the red-shifted $\mathrm{Ni}$ line, the filled circles correspond to the red-shifted Fe lines, and the open boxes correspond to the blue-shifted $\mathrm{Ni}$ line. The solid curves are the calculation assuming the mass of the compact object to be $30 \mathrm{M} \odot$, i.e., a black hole.

By the exploration in the parameter space, it was found that the length of the X-ray jet depends mostly on the initial density $n_{\mathrm{e} 0}$ and depends little on other parameters. Assuming a length of $l_{\mathrm{X}}=\mathrm{a}$ few $\times 10^{13} \mathrm{~cm}$, the density was estimated as $n_{\mathrm{e} 0}=\mathrm{a}$ few $\times 10^{12} \mathrm{~cm}^{-3}$. The model predicts that line luminosity is proportional to $\Theta^{-1} \dot{m}^{3 / 2} n_{\mathrm{e} 0}^{1 / 2}$ (Kotani et al. 1996a), thus we obtained the mass outflow rate of $\dot{m}=$ a few $\times 10^{6} \times\left(\Theta / 2.5^{\circ}\right) \mathrm{M} \odot \mathrm{yr}^{-1}$. When the precise $\mathrm{X}$-ray jet length is obtained with the tomography described in the next section, these physical quantities will be constrained with an accuracy never achieved previously.

\section{The Eclipse}

The temporal variation of X-ray flux enables to probe the geometry and the dimension of the system. The X-ray jet length is comparable to the sizes of other elements of the system, such as the orbital separation, the radius of the companion star and the accretion disk, thus can be a good measure of them. Since ASCA can resolve the "red" and "blue" components of the emission lines, we can observe the eclipse of each component separately (figure 2). occults which jet during eclipse. In figure 2 , emission line light curves covering an eclipse are plotted. The blue-shifted Fe lines show the clear eclipse lasting $\sim 2 \mathrm{dy}$, while the eclipse of the red Fe lines and the blue $\mathrm{Ni}$ line are not clear.

An (unsuccessful) example of calculated light curves are superimposed on figure 2. The calculation is based on a model with a geometrically thin bipolar jet and a spherical companion star. The jet has the thermal structure predicted by the conical plasma model. The companion was assumed to fill the Roche lobe. As the companion occults the jet, the flux of the emission lines decreases. The duration of the eclipse would determine the ratio of the companion radius 
to the orbital separation, and the depth of the eclipse would determine the ratio of the jet length to the companion radius. With the mass function of 2.0 $\mathrm{M} \odot$ reported by D'Odorico et al. (1991), the lobe size enables us to determine the absolute size of the companion radius, the orbital separation, the X-ray jet length, and the mass of the system. However, no parameters were found to satisfy the observation so far. The example in figure 2, assuming a $30 \mathrm{M} \odot$ black hole, predicts a too shallow and too short eclipse for the blue Fe lines. The assumption of a neutron star can not explain the shallow eclipse of the red $\mathrm{Fe}$ lines. The simple explanation is that something is wrong with the geometrical model. The companion may not be modeled as a Roche-lobe-filling sphere, or the jet may not be thin.

\section{Other Subjects}

The results presented here are only a fraction of the whole data and analysis. We had to omit to mention to the velocity of the X-ray-emitting region of the jet and the comparison of it with simultaneous optical observation (Kawai 1996; Kawai et al. 1996). Neither is there enough space to discuss on the precessional variation of the jet temperature, which tells us on the shape and kinematics of the disk. The results will be discussed and shown in another paper(s) in near future. ASCA surely progresses our understandings of SS 433 greatly and is expected to provide new picture of the system.

\section{References}

D’Odorico, S., Oosterloo, T., Zwitter, T., Calvani, M. 1991, Nature, 353, 329

Brinkmann, W., Kawai, N., Matsuoka, M., \& Fink, H. H. 1991, A\&A, 241, 112 Kawai N. 1996, in Multifrequency Behavior of High Energy Cosmic Sources, in press

Kawai, N., Kotani, T., Yamada, T., \& collaborators 1996, in Röntgenstrahlung from the Universe, H. U. Zimmermann, J. E. Trümper, \& H. Yorke, MaxPlanck-Institut für Extraterrstrische Physik: Garching, 153

Kotani, T., Kawai, N., Aoki, T., Doty, J., et al. 1994, PASJ, 46, L147

Kotani, T., Kawai, N., Matsuoka, M., \& Brinkmann, W. 1996a, PASJ, 48, 619

Kotani, T., Kawai, N., Matsuoka, M., \& Brinkmann, W. 1996b, in X-ray Imaging and Spectroscopy of Cosmic Hot Plasmas, F. Makino, Universal Academy Press: Tokyo, in press

Shapiro, P. R., Milgrom, M., Rees, M. J. 1986, ApJS, 60, 393

Stewart, G. C., Watson, M. G., Matsuoka, M., Brinkmann, W., et al. 1987, MNRAS, 228, 293

Tanaka, Y., Inoue, H., \& Holt, S. S. 1994, PASJ, 46, L37

Watson, M. G., Stewart, G. C., Brinkmann, W., \& King, A. R. 1986, MNRAS, 222,261 


\section{Discussion}

A. Konigl: It is interesting to note in connection with your finding that the $\mathrm{Ni}$ abundance is strongly enhanced in the jets that $\mathrm{Ni}$ overabundance was also inferred from Fabry-Perot observations of the optical filaments in the rim of the WSO shell surrounding the SS433 system (Mazek et al. 1982, ApJ). This might imply that the optically visible material in the filaments originates at least partially in the jets.

T. Kotani: That is surely important for the study of hydrodynamical evolution of the X-Ray lobes. My collaborators are now working on it. 